\title{
RINGS OF ANALYTIC FUNCTIONS
}

\section{JULIANNE SOUCHEK}

If $F$ is an open Riemann surface and $A(F)$ is the set of all analytic functions on $F$, then $A(F)$ is a ring under pointwise addition and multiplication. This paper is concerned with proper subrings $R$ of $A(F)$ which are isomorphic images of $A(G)$, the ring of all analytic functions on an open Riemann surface $G$, under a homomorphism $\Phi$ which maps constant functions onto themselves. The ring $R$ has the form $\{g \circ \phi$ : $g \in A(G), \phi$ an analytic map from $F$ into $G\}$, and will be denoted $R_{\phi}$. Relations between $\phi, R_{\phi}$ and the spectrum of $R_{\phi}$ are given as necessary and sufficient conditions for the existence of a Riemann surface $G$ such that $R$ is isomorphic to $A(G)$.

Open Riemann surfaces will be denoted by $F$ and $G$, the rings of all analytic functions on $F$ and $G$ with pointwise addition and multiplication will be denoted by $A(F)$ and $A(G)$, and $\Phi$ will denote a homomorphism from $A(G)$ into $A(F)$ which maps constant functions onto themselves. Let $\Phi$ be such a homomorphism. In [5, pp. 272273] H. L. Royden shows there is an analytic mapping $\phi$ of $F$ into $G$ such that $\Phi(g)=g \circ \phi$, and that if $\Phi$ is an isomorphism onto $A(F)$ then $\phi$ is a one-to-one, onto analytic mapping. If $\phi$ is an analytic mapping of $F$ into $G$, then $\Phi$ defined by $\Phi(g)=g \circ \phi, g \in A(G)$, is a homomorphism from $A(G)$ into $A(F)$ which preserves constant functions. When $\phi$ is one-to-one and onto, $\Phi$ is an isomorphism.

The image of $A(G)$ under $\Phi$ is the set $\{g \circ \phi: g \in A(G), \phi$ is an analytic map of $F$ into $G\}$ denoted by $R_{\phi}$. $\quad R_{\phi}$ is a subring of $A(F)$ and contains the constant functions, since $\Phi(\lambda)=\lambda$ for $\lambda$ a constant function. The following conditions are equivalent: $R_{\phi}$ properly contains the constant functions, $\Phi$ is an isomorphism, $\phi$ is not a constant function. Theorems 1 and 2 give other relations between $\phi$ and $R_{\phi}$.

THEOREM 1. If $R_{\phi}$ properly contains the constant functions, then $R_{\phi}$ contains $1 / f$ whenever $f \in R_{\phi}, f(z) \neq 0$ on $F$, if and only if $\phi$ maps $F$ onto $G$.

Proof. Let $\phi$ map $F$ onto $G, f \in R_{\phi}, f(z) \neq 0$ on $F$. Then $f=$ $\Phi h$ for some $h \in A(G)$ and $1 / h \in A(G)$ if $h(y) \neq 0$ for $y \in G$. Suppose $h(a)=0$. Since $a=\phi(z)$ for some $z \in F, 0=h(a)=h(\phi(z))=\Phi h(z)=$ $f(z)$. This contradicts $f(z) \neq 0$ on $F$. Thus $h(a) \neq 0$ for $a \in G$, $1 / h \in A(G)$, and $1 / f=\Phi(1 / h) \in R_{\phi}$.

Suppose $R_{\phi}$ contains $1 / f$ when $f \in R_{\phi}, f(z) \neq 0$ on $F$. Let $a \in G$. 
There is $g \in A(G)$ such that $g(a)=0$ and $g(w) \neq 0$ for $w \neq a[1$, pp. 591-592]. The function $\Phi g \in R_{\phi}$. If $\Phi g(z)=g \circ \phi(z) \neq 0$ for $z \in F$, then there is $h \in R_{\phi}$ such that $(\Phi g)(h)=1$. There is $k \in A(G)$ such that $h=\Phi k$. Then $(\Phi g)(\Phi k)=1$ and $\Phi(g k)=1$ but $\Phi$ is an isomorphism implies $g k=1$ and $g(a) k(a)=1$. This contradicts $g(a)=0$. Therefore $g(\phi(z))=0$ and $\phi(z)=a$ for some $z \in F$.

\section{A straightforward argument shows}

THEOREM 2. If $R_{\phi}$ properly contains the constant functions, then $R_{\phi}$ separates the points of $F$ if and only if $\phi$ is one-to-one.

Let $R$ be a ring of analytic functions defined on $F$. The spectrum of $R, \Sigma R$, is the set of nonzero homomorphisms $\pi$ from $R$ into the complex numbers such that $\pi(\lambda)=\lambda$ for $\lambda$ a constant function. For $x \in F$ the point evaluation mapping $\pi_{x}=\{(f, f(x)): f \in R\}$ is a homomorphism from $R$ into the complex numbers, and $\pi_{x}(\lambda)=\lambda$ for $\lambda$ a constant function. Therefore $\Sigma R$ always contains the point evaluation mappings defined on $R$. In [5, p. 272] H. L. Royden shows that the spectrum of $A(F)$ is the set of point evaluation mappings $\pi_{x}$ defined on $A(F), x \in F$. For $f \in R$ let $\hat{f}=\{(\pi, \pi f): \pi \in \Sigma R\} ; \hat{f}$ is a function from $\Sigma R$ into the complex numbers. Let $\hat{R}$ denote $\{\hat{f}: f \in R\}$. With pointwise addition and multiplication $\hat{R}$ is a ring containing the constant functions and is isomorphic to $R$ under $f \rightarrow \hat{f}$.

For $y \in G$, let $\psi_{y}$ denote an element of $\Sigma A(G)$. The mapping $P=\left\{\left(y, \psi_{y}\right): y \in G\right\}$ is a one-to-one function from $G$ onto $\Sigma A(G)$. If $R=\Phi(A(G))$ and $\Phi$ is an isomorphism, $L=\{(\pi, \pi \circ \Phi): \pi \in \Sigma R\}$ is a one-to-one function from $\Sigma R$ onto $\Sigma A(G)$. The mapping $\pi \rightarrow \pi \circ \Phi=$ $\psi_{y} \rightarrow y$ which is $P^{-1} \circ L$ defines a one-to-one correspondence between $\Sigma R$ and $G$ when $\Phi$ is an isomorphism.

THEOREM 3. Let $R_{\phi}=\Phi(A(G))$, $\Phi$ be an isomorphism from $A(G)$ into $A(F)$ which preserves constant functions. Let $M$ be the function from $\Sigma A(F)$ into $\Sigma R_{\phi}$ defined by $M\left(\pi_{x}\right)=\left.\pi_{x}\right|_{R_{\phi}}$. Then $M$ is onto if and only if $\phi$ is onto, and $M$ is one-to-one if and only if $\dot{ }$ is one-to-one.

Proof. The proof that $M$ is one-to-one if and only if $\phi$ is oneto-one follows from Theorem 2 and the fact that $A(F)$ separates the points of $F$.

Let $\pi \in \Sigma R_{\phi}$. Then $\pi \circ \Phi \in \Sigma A(G)$ implies there is $y \in G$ such that $\pi \circ \Phi=\psi_{y}$, where $\psi_{y}(g)=g(y)$ for $g \in A(G)$. There are two cases: $y \in \phi(F), y \notin \phi(F)$. If $y \in \phi(F)$, then $y=\phi(x)$ for some $x \in F$ and $\pi(\Phi g)=g(y)=g(\phi(x))=\Phi g(x)$ for every $g \in A(G), \pi(\Phi g)=\Phi g(x)$ for 
every $f=\Phi g \in R_{\phi}$. This implies $\pi=M\left(\pi_{x}\right)$. If $y \notin \phi(F)$, then $y \neq \phi(x)$ for $x \in F$, and it may be shown that for every $x \in F$ there is $f \in R_{\phi}$ such that $\pi(f) \neq f(x)$. Let $x \in F$. Then $\phi(x) \in G . \quad y \in G, \quad y \neq \phi(x)$, and $A(G)$ separates the points of $G$ implies there is a $g \in A(G)$ such that $g(y) \neq g(\phi(x))$. From $\Phi(g) \in R_{\phi}$ and $\pi(\Phi g)=g(y) \neq g(\phi(x))=\Phi g(x)$ it follows that $\pi \neq M\left(\pi_{x}\right)=\left.\pi_{x}\right|_{R_{\dot{\phi}}}$.

For $\pi \in \Sigma R_{\phi}, \pi \circ \Phi=\psi_{y} \in \Sigma A(G)$, and it has been shown $\pi \in M(\Sigma A(F))$ if and only if $y \in \phi(F)$.

From Theorem 3 and since $\Sigma R_{\phi}$ and $G$ are in one-to-one correspondence, it follows that the point evaluation maps in $\Sigma R_{\phi}$ are in one-to-one correspondence with the points $\phi(x) \in \phi(F)$, and the elements of $\Sigma R_{\phi}$ which are not point evaluation maps are in one-to-one correspondence with the points in $G-\phi(F)$.

Theorem 4 contains a necessary condition which a subring $R$ of $A(F)$ must satisfy if $R$ is to be $\Phi(A(G))$, the isomorphic image of $A(G)$ under $\Phi$ for some open Riemann surface $G$. The corollary to Theorem 5 gives a set of sufficient conditions on $R$ in order that $R$ be $\Phi(A(G))$ when $\Phi g=g \circ \phi$ and $\phi: F \rightarrow G$ is an onto mapping.

Suppose $F$ is an open Riemann surface, $p \in F, f$ is analytic at $p$ and $\tau$ is a local uniformizer which maps a neighborhood of $p$ onto $\{z:|z|<g\}$ for some $\rho>0, \tau(p)=0$. There is a number $r>0$ such that $f \circ \tau^{-1}(z)=\sum_{i=0}^{\infty} a_{i} z^{i}$ for $|z|<r$. The multiplicity of $f$ at $p$ is defined as $\inf \left\{k: k \neq 0\right.$ and $\left.a_{k} \neq 0\right\}$, denoted $n(p ; f)$. The multiplicity $n(p ; f)$ of $f$ at $p$ does not depend on $\tau$. If $R$ contains functions other than constants, $m=\inf \{n(p ; f): f \in R\}$ is defined, and $n(p ; f)=m$ for some $f \in R$.

THEOREM 4. Let $p \in F, R_{\dot{\phi}}$ contain functions other than constants and let $m=\left\{\inf n(p ; f): f \in R_{\phi}\right\}$. There is a local uniformizer $\tau$ at $p$ with the properties: $\tau(0)=p$, for some $\rho>0, \tau$ maps $\{z$ : $|z|<\rho\}$ onto a neighborhood of $p$, and if $f \in R_{\phi}, f \circ \tau(z)=\sum_{i=0}^{\infty} a_{i}\left(z^{m}\right)^{i}$ for $|z|<\rho$.

The proof of Theorem 4 is based on two lemmas:

Lemma 1. If $p \in F, m=\inf \left\{n(p ; f): f \in R_{\phi}\right\}$ and $f \in R_{\phi}$, then $n(p ; f)=k m$, where $k$ is a positive integer.

Lemma 2. Given $\sum_{i=m}^{\infty} c_{i} z^{i}$ convergent for $|z|<\rho, c_{m} \neq 0, m \neq 0$, there is $\sum_{i=1}^{\infty} b_{i} z^{i}$ convergent for $|z|<\rho, b_{1} \neq 0$, such that $\left(\sum_{i=1}^{\infty} b_{i} z^{i}\right)^{m}=$ $\sum_{i=m}^{\infty} c_{i} z^{i}$.

Lemma 1 follows from the two relations: For $f \in R_{\dot{\phi}}, f=g \circ \phi$ for 
some $g \in A(G)$, which implies $n(p ; f)=(n(p ; \phi))(n(\phi(p) ; g))$, and if $m=$ $\inf \left\{n(p ; f): f \in R_{\phi}\right\}$ then $n(p ; \phi)=m$. Lemma 2 is proved by defining $W$ a subset of the natural numbers $N$ as $W=\left\{n \in N: b_{1}, b_{2}, \cdots, b_{n}\right.$ can be defined in such a way that the coefficients of $z^{i}$ for $1 \leqq m \leqq$ $i \leqq m+n-1$ of $\left(\sum_{i=1}^{\infty} b_{i} z^{i}\right)^{m}$ and $\sum_{i=m}^{\infty} c_{i} z^{i}$ are equal $\}$ and using induction to show $W=N$.

Proof of Theorem 4. Let $\tau_{p}$ be a local uniformizer about $p$ such that $\tau_{p}(0)=p$. If $m=\inf \left\{n(p ; f): f \in R_{\phi}\right\}$, there is $f_{p} \in R_{\phi}$ and $\rho>0$ such that $f_{p} \circ \tau_{p}(z)=\sum_{i=m}^{\infty} c_{i} z^{i}$ for $|z|<\rho, c_{m} \neq 0$, and the range of $\sum_{i=m}^{\infty} c_{i} z^{i}$ contains $|z|<\rho^{m}$.

There is a power series $\sum_{i=1}^{\infty} b_{i} z^{i}, b_{1}^{m}=c_{m}$, such that $\sum_{i=m}^{\infty} c_{i} z^{i}=$ $\left(\sum_{i=1}^{\infty} b_{i} z^{i}\right)^{m}$ for $|z|<\rho$ as stated in Lemma 2. $k(z)=\sum_{i=1}^{\infty} b_{i} z^{i}$ is defined for $|z|<\rho$, is one-to-one, and its range contains $|z|<\rho$. Thus $k^{-1}(y)$ is defined for $|y|<\rho$ and $f_{p} \circ \tau_{p} \circ k^{-1}(z)=\left(\sum_{i=1}^{\infty} b_{i}\left(k^{-1}(z)\right)^{i}\right)^{m}=$ $z^{m}$ for $|z|<\rho, \tau_{p} \circ k^{-1}(0)=p$. The function $\tau=\tau_{r} \circ k^{-1}$ is a local uniformizer about $p$ and there is $f_{p} \in R_{\phi}$ such that $f_{p} \circ \tau(z)=z^{m}$ for $|z|<\rho$.

Let $f \in R_{\phi}, f$ not a constant function. Then $f \circ \tau(z)=\sum_{i=0}^{\infty} a_{i} z^{i}$ for $|z|<\rho$. Let $N$ denote the natural numbers and define $W=$ $\left\{n \in N: f \circ \tau(z)=\sum_{i=0}^{n} a_{m j_{i}} z^{m j_{i}}+z^{m j_{n}} h_{n}(z)\right.$, where $h_{n}(z)=\sum_{i=1}^{\infty} b_{n, i} z^{i}$ and $j_{i}$ are nonnegative integers, $\left.0=j_{0}<j_{1}<\cdots<j_{n}\right\}$.

It follows from Lemma 1 that for $|z|<\rho, f \circ \tau(z)=\sum_{i=0}^{\infty} a_{i} z^{i}=$ $a_{0}+a_{m j_{1}} z^{m j_{1}}+z^{m j_{1}} h_{1}(z)$, where $h_{1}(0)=0$. If $k \in W$, then $f \circ \tau(z)=$ $\sum_{i=0}^{k} a_{m j_{i}} z^{m j_{i}}+z^{m j_{k}} h_{k}(z), h_{k}(0)=0$. Since $f \in R_{\dot{\phi}}, z^{m} \in R_{\phi}$ and constants are contained in $R_{\phi}, z^{m j_{k}} h_{k}(z)=f(z)-\sum_{i=0}^{k} a_{m j_{j}} z^{m j_{i}} \in R_{\phi}$. If $h_{k} \neq 0$, $n\left(p ; z^{m j_{k}} h_{k}\right)=m j_{k+1}$ and $f \circ \tau(z)=\sum_{i=0}^{k+1} a_{m j_{i}} z^{m j_{i}}+z^{m j_{k+1}} h_{k+1}(z)$, where $h_{k+1}(z)=\sum_{i=1}^{\infty} b_{k+1, i} z^{i}$ on $|z|<\rho$ and $j_{k+1}>j_{k}$. If $h_{k}=0$, then the above statement is true with $a_{m j_{k+1}}=0, h_{k+1}=0$. By induction $W=N$ and $f \circ \tau(z)=\sum_{i=0}^{\infty} a_{m i} z^{m i}$ on $|z|<\rho$.

If $R$, a subring of $A(F)$, has the property that for every $a \in F$, $f \in R$, for some local uniformizer $\tau$ about $a, f \circ \tau(z)=\sum_{i=0}^{\infty} a_{i}\left(z^{m(a)}\right)^{i}$ for $m(a)=\inf \{n(a ; f): f \in R\}$, then $R$ has property $(\xi)$. If $R$ contains functions other than constants and has property $(\xi)$, then for $a \in F$, $m(a)=\inf \{n(a ; f): f \in R\}=1$ if $R$ separates the points of $F$.

THEOREM 5. If $R$ is a subring of $A(F)$ which contains func-

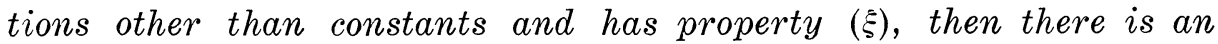
open Riemann surface $G$, an analytic mapping $\phi$ of $F$ onto $G$, and a separating subring $S$ of $A(G)$ such that $S$ is isomorphic to $R$ under $\hat{f} \rightarrow \hat{f} \circ \phi, \hat{f} \in S .{ }^{1}$

Proof. Let $G=\left\{\pi_{p}: p \in F\right\}$ where $\pi_{p}=\{(f, f(p)): f \in R\}$ and $\phi=$ 
$\left\{\left(p, \pi_{p}\right): p \in F\right\}$. The topology on $G$ will be that which makes $\phi$ continuous and open. If $N_{p}$ is an open neighborhood of $p \in F$, then $N_{\pi_{p}}=\left\{\pi_{q}: q \in N_{p}\right\}$ is an open neighborhood of $\pi_{p}$. The set $G$ with this topology is a connected Hausdorff space.

Let $p \in F, \pi_{p} \in G$ and $m=\inf \{n(p ; f): f \in R\}$. By the same argument used in the beginning of the proof of Theorem 4, there is a function $f_{p} \in R$ and a local uniformizer $\tau$ about $p$ such that $\tau(0)=p$ and $f_{p} \circ \tau(z)=z^{m}$ for $|z|<\rho^{1 / m}$ for some $\rho>0$. Then for $f \in R$, $f \circ \tau(z)=\sum_{i=0}^{\infty} a_{i}\left(z^{m}\right)^{i}=g_{f}\left(z^{m}\right)$ for $|z|<\rho^{1 / m}, g_{f}$ analytic on $|z|<\rho$.

It will be shown that $\sigma_{\tau}=\left\{\left(z^{m}, \pi_{\tau(z)}\right):|z|<\rho^{1 / m}\right\}$ is a local uniformizer about $\pi_{p}$. If $z_{1}^{m}=z_{2}^{m}$, then $f \circ \tau\left(z_{1}\right)=g_{f}\left(z_{1}^{m}\right)=g_{f}\left(z_{2}^{m}\right)=$ $f \circ \tau\left(z_{2}\right)$, for $f \in R$ implies $\pi_{\tau\left(z_{1}\right)}=\pi_{\tau\left(z_{2}\right)}$, which implies that $\sigma_{\tau}$ is a function. If $\pi_{\tau\left(z_{1}\right)}=\pi_{\tau\left(z_{2}\right)}$ then in particular $f_{p} \circ \tau\left(z_{1}\right)=f_{p} \circ \tau\left(z_{2}\right)$, which implies $z_{1}^{m}=z_{2}^{m}$, and $\sigma_{\tau}$ is one-to-one. Since the relations $z^{m} \rightarrow z \rightarrow$ $\tau(z) \rightarrow \phi(\tau(z))=\pi_{\tau(z)}$ are open and continuous, $\sigma_{\tau}$ is open and continuous. Thus $\sigma_{z}$ is a homeomorphism from $\{w:|w|<\rho\}$ onto $\phi \circ \tau\left(\left\{z:|z|<\rho^{1 / m}\right\}\right)=$ $N_{\pi_{p}}$.

If $\pi \in W=\sigma_{\tau_{2}}\left(|z|<\rho_{2}\right) \cap \sigma_{\tau_{1}}\left(|z|<\rho_{1}\right)$, there are points $z_{1}, z_{2}$ such that $\pi_{\tau_{1}}\left(z_{1}\right)=\pi_{\tau_{2}}\left(z_{2}\right)$. Then $f \circ \tau_{1}\left(z_{1}\right)=f \circ \tau_{2}\left(z_{2}\right)$ for every $f \in R$, and $z_{1}^{m_{1}}=$ $f_{1}\left(\tau_{1}\left(z_{1}\right)\right)=f_{1}\left(\tau_{2}\left(z_{2}\right)\right)=g_{f_{1}}\left(z_{2}^{m_{2}}\right)$, so $g_{f_{1}}$ is analytic on $\left\{w:|w|<\rho_{2}\right\}$, which contains $\sigma_{\tau_{2}}^{-1}(W)$. This shows that $z_{1}^{m_{1}}=\sigma_{\tau_{1}}^{-1} \circ \sigma_{\tau_{2}}\left(z_{2}^{m_{2}}\right)$ is analytic on $\sigma_{\tau_{2}}^{-1}(W)$ to $\sigma_{\tau_{1}}^{-1}(W)$. The function $\sigma_{\tau}$ is a local uniformizer of a neighborhood of $\pi_{p}$, and $G$ is a Riemann surface.

For $f \in R$, let $\hat{f}=\left\{\left(\pi_{p}, f(p)\right): p \in F\right\}, S=\{\hat{f}: f \in R\}$. Since $f$ is continuous and $\phi$ is open, $\hat{f}$ is continuous. The function $\hat{f}$ is analytic at $\pi_{p}$, because if $|w|<\rho, w=z^{m}$, then $\hat{f}_{\circ} \sigma_{\tau}(w)=\hat{f}\left(\pi_{\tau(z)}\right)=f(\tau(z))=$ $\sum_{i=0}^{\infty} a_{i}\left(z^{m}\right)^{i}=\sum_{i=0}^{\infty} a_{i} w^{i}$. The mapping $\phi$ is analytic at $p$, because $\sigma_{z}^{-1} \circ \phi \circ \tau(z)=\sigma_{\tau}^{-1}\left(\pi_{\tau(z)}\right)=z^{m}$ for $|z|<\rho^{1 / m}$. With pointwise addition and multiplication, $S$ is a ring and is isomorphic to $R$ under the mapping $\hat{f} \rightarrow \hat{f} \circ \dot{\phi}=f$. The ring $S$ separates the points of $G$. Since $S$ contains functions which are not constant and are analytic on $G, G$ is an open Riemann surface.

If $S$ is to be $A(G)$, then by Theorem 3 the mapping $M\left(\pi_{p}\right)=\left.\pi_{p}\right|_{R}$ from $\Sigma A(F)$ to $\Sigma R$ must be onto, since $\phi$ is an onto mapping of $F$ to $G$. Thus $\Sigma R$ may contain only point evaluation mappings and $\Sigma R=G$.

Corollary to Theorem 5. If $R$ is a subring of $A(F)$ which properly contains the constant functions and has property ( $\xi)$, if $\Sigma R$ contains only point evaluation mappings, and $R$ contains all $f \in A(F)$ such that $f \circ \tau_{p}(z)=\sum_{i=0}^{\infty} a_{i}\left(z^{m}\right)^{i}$ for $|z|<\rho^{1 / m}, \quad p \in F, \quad m=$ $\inf \{n(p: f): f \in R\}$, then $\Sigma R=G$ is an open Riemann surface, and $R$ is isomorphic to $S=A(G)$.

1 This result and proof are similar to one given by M. Heins for a subfield of the field of all meromorphic functions on a Riemann surface [2, pp. 268-269]. 
Proof. Everything except $S=A(G)$ was shown in the proof of Theorem 5. The function $\hat{f} \in A(G)$ if and only if for every $\pi_{p} \in G$, $\hat{f}_{\circ} \sigma_{\bar{s}_{p}}(w)=\sum_{i=0}^{\infty} a_{i} w^{i}$ for $|w|<\rho$. Let $\hat{f} \in A(G), p \in F, \pi_{p} \in G$, and $f=\hat{f} \circ \dot{\phi}$. Then $f \in A(F)$ and $f \in R$, because for $|z|<\rho^{1 / m}, f \circ \tau_{p}(z)=$ $\hat{f}_{\circ} \phi\left(\tau_{p}(z)\right)=\hat{f}\left(\pi_{\tau_{p}(z)}\right)=\hat{f} \circ \sigma_{\tau_{p}}\left(z^{m}\right)=\sum_{i=0}^{\infty} a_{i}\left(z^{m}\right)^{i}$.

If $R=\{\hat{f} \circ \phi: \hat{f} \in S\}$ and $S$ separates the points of $G$, then $R$ separates the points of $F$ if and only if $\phi$ is a one-to-one function. If $S$ separates the points of $G$, and $S=A(G)$, then $R$ may not separate the points of $F$, because if it did $\phi$ would be a one-to-one, onto analytic function from $F$ to $G$, and $R=A(F)$. If $S \neq A(G)$ there may be a surface $H$, a mapping $\phi_{1}$ and a separating subring $T$ of $A(H)$ such that $\phi_{1}$ is analytic and one-to-one but not onto, and $T=$ $A(H)$.

In this part of the paper it is noted that if $R=\Phi(A(G))$, then $\Sigma R$ with the Gelfand topology is an open Riemann surface, and $\hat{R}$ which is isomorphic to $R$, is the ring of all analytic functions on $\Sigma R$. Theorem 8 gives sufficient conditions on a subring $R$ of $A(F)$ and on $\hat{R}$ in order that $\Sigma R$ be an open Riemann surface and $\hat{R}$ be a ring of analytic functions on $\Sigma R$. In conclusion sufficient conditions for $\hat{R}$ to be $A(\Sigma R)$ are given.

If $R$ is a ring of complex valued functions on $F$, then the Gelfand topology on $\Sigma R$ is the weakest topology on $\Sigma R$ which makes each element of $\hat{R}$ continuous, where $\hat{R}=\{\hat{f}: f \in R\}, \hat{f}=\{(\pi, \pi f): \pi \in \Sigma R\}$. Let $\pi_{0} \in \Sigma R, K$ be a finite subset of $\hat{R}, \varepsilon>0$. An open neighborhood of $\pi_{0}$ will be $\left\{\pi \in \Sigma R:\left|\hat{f}(\pi)-\hat{f}\left(\pi_{0}\right)\right|<\varepsilon\right.$ for $\left.\hat{f} \in K\right\}$. If $R=\Phi(A(G))$ and $\Phi$ is an isomorphism, then $\Sigma R$ and $\Sigma A(G)$ with the Gelfand topology are homeomorphic under the mapping $L(\pi)=\pi \circ \Phi$ from $\Sigma R$ onto $\Sigma A(G)$. The mapping $P(y)=\psi_{y}$ from $G$ onto $\Sigma A(G)$ with the Gelfand topology is one-to-one, onto and continuous. The mapping $P$ is also open. As Royden observes [4, pp. 287-288], this is a consequence of a theorem of Remmert that an open Riemann surface can be mapped one-to-one and holomorphically into $C^{3}[3$, p. 118]. Thus $P^{-1} \circ L$ is a homeomorphism from $\Sigma R$ with the Gelfand topology onto $G$.

THEOREM 6. If $R$ is a subring of $A(F)$ such that $R=\Phi(A(G))$, and if $\Phi$ is an isomorphism which preserves constant functions, then $\Sigma R$ with the Gelfand topology is an open Riemann surface, and $\hat{R}$ is the ring of all analytic functions on $\Sigma R$. Moreover $\hat{R}$ is isomorphic to $R$.

Proof. The spectrum of $R$ with the Gelfand topology is a Hausdorff space. It is homeomorphic to $G$ under the mapping $L^{-1} \circ P$, 
and is connected. Let $\pi_{q} \in \Sigma R$ where $q \in G, \psi_{q} \in \Sigma A(G)$, and $L^{-1} \circ P$ maps $q \rightarrow \psi_{q} \rightarrow \pi_{q}$. If $N_{q}$ is a neighborhood of $q$ then $N_{\pi_{q}}=L^{-1} \circ P\left(N_{q}\right)$ is a neighborhood of $\pi_{q}$. There exists $h_{q} \in A(G)$ which has a simple zero at $q$ [1, pp. 591-592]. $\quad h_{q}$ is a local uniformizer on a neighborhood of $q, \quad N_{q}=h_{q}^{-1}(|z|<\rho)$ for some $\rho>0$. If $\sigma_{q}=\left.h_{q}\right|_{N_{q}}$, then $h_{q} \circ \sigma_{q}^{-1}(z)=z$ for $|z|<\rho$. For $h \in A(G), y \in N_{q}, h(y)=\sum_{i=0}^{\infty} a_{i}\left(h_{q}(y)\right)^{i}$.

If $f_{q}=\Phi h_{q}$ then $\hat{f}_{q}$ is a local uniformizer on $N_{\pi_{q}}=L^{-1} \circ P\left(N_{q}\right)$. From $\hat{f}_{q}\left(\pi_{y}\right)=h_{q}(y)$ follows $\hat{f}_{q}\left(\pi_{y}\right)=h_{q} \circ P^{-1} \circ L\left(\pi_{y}\right), \pi_{y} \in N_{\pi_{q}}$, which implies $\hat{f}_{q}$ is a homeomorphism of $N_{\pi_{q}}$ onto $|z|<\rho$. If $\pi_{y} \in N_{\pi_{q_{1}}} \cap N_{\pi_{q_{2}}}$, then $\hat{f}_{q_{1}}\left(\pi_{y}\right)=h_{q_{1}}(y)=\sum_{i=0}^{\infty} a_{i}\left(h_{q_{2}}(y)\right)^{i}=\sum_{i=0}^{\infty} a_{i}\left(\hat{f}_{q_{2}}\left(\pi_{y}\right)\right)^{i}$ since $\pi_{y} \in N_{\pi_{q_{2}}}$ or $y \in N_{q_{2}}$. The function $\hat{f}_{q}$ is a local uniformizer on $N_{\pi_{q}}$ and $\Sigma R$ is a Riemann surface.

The ring $\hat{R}$ is contained in $A(\Sigma R)$, because if $\hat{f} \in \hat{R}, \pi_{y} \in N_{\pi_{q}}$, $z=\hat{f}_{q}\left(\pi_{y}\right)$, then $\hat{f}_{0} \hat{f}_{q}^{-1}(z)=\hat{f}\left(\pi_{y}\right)=h(y)=\sum_{i=0}^{\infty} a_{i}\left(h_{q}(y)\right)^{i}=\sum_{i=0}^{\infty} a_{i}\left(\hat{f}_{q}\left(\pi_{y}\right)\right)^{i}=$ $\sum_{i=0}^{\infty} a_{i} z^{i}$. The function $T(q)=\pi_{q}$ is an analytic map of $G$ onto $\Sigma R$. If $\theta$ is analytic on $\Sigma R$, then $\theta \circ T \in A(G)$ and $\theta \in \hat{R}$ because $\theta\left(\pi_{q}\right)=$ $\theta \circ T(q)=\psi_{q}(\theta \circ T)=\pi_{q}(f)$ for $f=\Phi(\theta \circ T)$. This implies $\theta=\hat{f}$. Thus $\hat{R}=A(\Sigma R)$. Since $\hat{R}$ contains functions which are analytic and are not constant on $\Sigma R, \Sigma R$ is an open Riemann surface.

Theorem 7. Let $R=\Phi(A(G))$. If $\hat{\pi} \in \Sigma R$, then $\hat{\pi}^{-1}(0)$ is a principal maximal ideal of $R$, and every principal maximal ideal of $R$ is the kernel of $\pi \in \Sigma R$. If $\hat{\pi}^{-1}(0)$ is generated by $f$, then $\hat{f}$ is a local homeomorphism on a neighborhood $N_{\hat{\pi}}$ of $\hat{\pi}$ and if $\pi \in N_{\hat{\pi}}$, $\hat{k} \in \hat{R}$, then $\hat{k}(\pi)=\sum_{i=0}^{\infty} a_{i}(\hat{f}(\pi))^{i}$.

Proof. If $\hat{\pi} \in \Sigma R$, then $\hat{\pi} \circ \Phi=\psi_{q} \in \Sigma A(G)$ and $\hat{\pi}^{-1}(0)=\Phi\left(\psi_{q}^{-1}(0)\right)$. The kernel of $\psi_{q}, M_{q}=\psi_{q}^{-1}(0)$, is a principal maximal ideal of $A(G)$, and every principal maximal ideal of $A(G)$ is a kernel of $\psi \in \Sigma A(G)$ [5, pp. 271-272]. If $h$ generates $M_{q}$, then $h$ has a single zero and it is a simple zero at $q$ [5]. Thus $h$ is a homeomorphism on a neighborhood of $q, N_{q}$. If $f=\Phi h$, then $\hat{\pi}^{-1}(0)$ is the ideal generated by $f$. Also $\hat{f}$ is a uniformizer on $N_{\hat{\pi}}=L^{-1} \circ P\left(N_{q}\right)$, and if $\pi \in N_{\hat{\pi}}, \hat{k} \in \hat{R}$, then $\hat{k}(\pi)=\sum_{i=0}^{\infty} a_{i}(\hat{f}(\pi))^{i}$ as shown in the proof of Theorem 6 .

Lemma. Let $S$ be a ring of continuous functions on $X$ with identity. Then $X$ is not connected if and only if $S$ is contained in a ring $Q$ of continuous functions on $X$, where $Q=I_{1}+I_{2}, I_{1}, I_{2}$ proper ideals of $Q, I_{1} \cap I_{2}=\{0\}$.

THEOREM 8. Let $R$ be a subring of $A(F)$ which properly contains the constant functions, and suppose $\hat{R}$ is not contained in a ring $Q$ of continuous functions on $\Sigma R$ where $Q=I_{1}+I_{2}, I_{1}, I_{2}$ proper ideals of $Q, I_{1} \cap I_{2}=\{0\}$. If for $\hat{\pi} \in \Sigma R, \hat{\pi}^{-1}(0)$ is a principal ideal of $R$ 
generated by $f$ and $\hat{f}$, the function in $\hat{R}$ which corresponds to $f$ in $R$, is a homeomorphism on a neighborhood of $\hat{\pi}$, and for $\pi$ in this neighborhood, $g \in R, \pi g=\sum_{i=0}^{\infty} a_{i}(\pi f)^{i}$, then $\Sigma R$ is an open Riemann surface and $\hat{R}$ is a ring of analytic functions on $\Sigma R$.

Proof. The spectrum of $R$ with the Gelfand topology is a Hausdorff space. By the lemma $\Sigma R$ is connected. Let $\hat{\pi} \in \Sigma R$. There is $\hat{f}$ a homeomorphism of $N_{\hat{\pi}}$ onto $|z|<\rho$ for some $\rho>0$. If $\pi \in N_{\hat{\pi}}$, $g \in R$, then $\widehat{g}(\pi)=\sum_{i=0}^{\infty} a_{i}(\hat{f}(\pi))^{i}$. If $\pi \in N_{\pi_{1}} \cap N_{\pi_{2}}=W$ then $\hat{f}_{1} \circ \hat{f}_{2}^{-1}\left(\hat{f}_{2}(\pi)\right)=$ $\hat{f}_{1}(\pi)=\sum_{i=0}^{\infty} a_{i}\left(\hat{f}_{2}(\pi)\right)^{i}$ implies $\hat{f}_{1}^{\circ} \circ \hat{f}_{2}^{-1}$ is analytic on $\hat{f}_{2}(W)$. $\left\{\left(N_{\pi}, \hat{f}_{\pi}\right)\right.$ : $\pi \in \Sigma R\}$ defines an analytic structure on $\Sigma R$. It is immediate that $\hat{R} \subset A(\Sigma R)$. Since $\hat{R}$ contains functions which are not constant and are analytic on $\Sigma R, \Sigma R$ is an open Riemann surface.

If $\left\{R_{n}\right\}$ is a sequence of subrings of $A(F)$ such that $R_{n}$ satisfies the conditions of Theorem 8, $\left.\Sigma R_{n}\right|_{R_{1}}=\Sigma R_{1}, R_{n-1} \subset R_{n}$, then the chain has a maximal element, $\left\{\hat{f}_{\circ} \phi: \hat{f} \in A\left(\Sigma R_{1}\right)\right.$ and $\left.\phi(x)=\pi_{x}, x \in F\right\}$. Let $\hat{\pi} \in \Sigma R_{1}$ and $\hat{f}$ be a local homeomorphism at $\hat{\pi}$. If $R_{1}$ satisfies the conditions of Theorem 8 and contains all functions $g$ in $A(F)$ such that $\hat{g}(\pi)=\sum_{i=0}^{\infty} a_{i}(\hat{f}(\pi))^{i}$ for $\pi \in N_{\hat{\pi}}, \pi$ and $\hat{\pi}$ elements of $\Sigma R_{1}$, then $\hat{R}_{1}=A\left(\Sigma R_{1}\right)$, because if $\hat{g} \notin \hat{R}_{1}$, then there is $\hat{\pi} \in \Sigma R_{1}$ such that $\hat{g}_{\circ} \hat{f}^{-1}$ is not analytic on $\{z:|z|<\rho\}$ which implies $\widehat{g} \notin A\left(\Sigma R_{1}\right)$.

The author wishes to thank Professors H. B. Curtis and H. E. Lacey for their suggestions and encouragement.

\section{REFERENCES}

1. H. Behnke, and F. Sommer, Theorie der analytischen Funktionen einer komplexen Veränderlichen, Springer-Verlag, Berlin, 1962.

2. M. Heins, Algebraic structure and conformal mapping, Trans. Amer. Math. Soc. 89 (1958), 267-276.

3. R. Remmert, Sur les espaces analytiques holomorphiquement separables et holomorphiquement convexes, C. R. Acad. Sci. Paris 243 (1956), 118-121.

4. H. Royden, Function algebras, Bull. Amer. Math. Soc. 69 (1963), 281-298.

5. - Rings of analytic and meromorphic functions, Trans. Amer. Math. Soc. 83 (1956), 269-276.

Received July 24, 1968, and in revised form October 14, 1969. This paper is part of the author's Ph. D. thesis submitted to the University of Texas at Austin.

Sam Houston State University

HuntSVille, Texas 